\title{
ACTIVIDADES EXTRAESCOLARES EN MATEMÁTICAS PARA MEJORAR EL CÁLCULO MENTAL EN NIÑOS DE 7 AÑOS.
}

\author{
AFTER SCHOOL ACTIVITY IN MATHEMATICS TO IMPROVE MENTAL \\ CALCULATION IN CHILDREN AGED 7 YEARS.
}

\begin{abstract}
Patricia Álvarez
Universidad Técnica de Ambato, Av. Los Chasquis y Río Payamino, Campus Huachi, Ambato-Ecuador. Email:elizabethalvarezviera@outlook.es

https://doi.org/10.33789/talentos.7.2.132
\end{abstract}

\begin{abstract}
Resumen: Las actividades extraescolares en matemáticas surgen como una necesidad de ayudar a niños que no alcanzan las competencias, a mejorar su desempeño en esta área. El objetivo del estudio fue realizar varias actividades extraescolares con el fin de mejorar el cálculo mental en niños de 7 años. Se seleccionaron 14 niños, 9 niñas y 5 niños de 7 años. La intervención se efectuó en la Fundación Caminos del Sol durante 10 semanas, por dos horas por semana, durante los meses de mayo a julio de 2018. Se efectuaron actividades de repaso de tablas de sumas y restas, resolución de problemas de sumas, restas, combinación de sumas y restas y en cálculo mental. Los resultados determinaron que la intervención educativa mejoró el cálculo mental en los niños en un $80 \%$ más en el Postest con relación al Pretest. En conclusión, las actividades extraescolares en matemáticas ayudaron al desempeño de los niños en el cálculo mental.
\end{abstract}

Palabras clave: Aritmética mental, enseñanza de las matemáticas, pensamiento abstracto.

\begin{abstract}
After school mathematical activities are necessary to help children to improve their development in this area when are not doing well to achieve the competences needed. The objective of the study was to carry out several extracurricular activities in mathematics in order to improve mental calculation in 7-year-old. 14 children aged 7 years, 9 girls and 5 boys were chosen. The intervention was done at the Caminos del Sol Foundation for 10 weeks, for two hours each one, from May to July in 2018. Students worked on activities related to addition and
\end{abstract}

Recibido: 13 de noviembre de 2019

Aceptado: 9 de septiembre de 2020

Publicado como artículo científico en la Revista de Investigación Talentos VII (2), 24-32 
subtraction tables, solving problems of addition and subtraction, combination of addition and subtraction, and mental calculation. The results determined that the educational intervention improved the mental calculation about $80 \%$ of knowledge more in the Posttest than the Pretest. In conclusion, extracurricular activities in mathematics helped children's performance in mental calculation.

Keywords: Mental arithmetic, mathematics education, abstract thinking.

\section{INTRODUCCIÓN}

Las actividades extraescolares se realizan fuera del entorno escolar, están dirigidas a niños y jóvenes en actividades deportivas, programas artísticos, o refuerzos de materias escolares, quetienen comoobjetivomejorarlos rendimientos de los que participan (Gonzales, 2016). Las actividades extraescolares en el área de las matemáticas, surge como una necesidad de apoyar a los alumnos que no alcanzan las competencias básicas o presentan dificultades de aprendizaje en esta materia; problema que afecta el desarrollo normal en las aulas, con la consiguiente baja autoestima del estudiante y de sus padres.

En Ecuador, los niños de las escuelas se hallan en los puestos medios con respecto a las matemáticas cuando se compara con otros niños de la misma edad y de otros países de Sudamérica (Flotts et al., 2016), en otras palabras, los niños del país tienen dificultades a modo general para entender las matemáticas

Por otro lado, la enseñanza-aprendizaje de las matemáticas en niños tienen en esencia la aritmética y la geometría, por importancia en el cálculo y el desarrollo cognitivo de los alumnos (Poll y Guirado, 2017).
Las investigaciones neurocientíficas han permitido revelar que los niños en la etapa de educación infantil muestran un sentido numérico que les faculta adentrarse en el campo de la aritmética, sin que se le hayan enseñado el lenguaje simbólico asociado a este campo (Guillen, 2018).

Sin embargo, en el niño el número surge de las percepciones de ver, tocar, experimentar y manejar objetos (Moya, 2004), mientras más oportunidades tengan los niños para contar, mayor confianza y sentido tendrán con las matemáticas en las aulas (Oficina de Educación del Condado, 2010). Varios estudios han demostrado una correlación entre la resolución de problemas de matemáticas y la memoria de trabajo, que permite al niño almacenar y manipular temporalmente la información para la resolución de problemas, y también mencionan que entrenar la memoria de trabajo mediante ejercicios específicos mejoran el desempeño matemático (González, Fernández y Duarte, 2018).

Por lo que, es apropiado que el docente emplee estrategias didácticas en la enseñanza de las matemáticas por su importancia en la educación, para ello, tendrá que proponer métodos o técnicas o recursos que le sean 
fácil de emplear con el objetivo de mejorar en el alumno el aprendizaje en la resolución de problemas matemáticos de forma correcta y rápida (Melquiades, 2012).

Adicionalmente, el profesor de matemáticas debe enfocarse en promover el desarrollo del pensamiento matemático $\mathrm{y}$ las habilidades de razonamiento, debido a que el niño requiere una comprensión de la asociación e interrelación entre objetos, elementos, símbolos numéricos, cantidades y características (Parviainen, 2019); la finalidad es que conozca y dominen el lenguaje matemático que le permita razonar, usar la argumentación, la prueba y la refutación en el desarrollo del cálculo (Penagos, Mariño y Hernández, 2017).

Además, para que el niño aprenda operaciones aritméticas, se enseña a memorizar y repetir las tablas de sumas, restas y multiplicación (González y Arencibia, 2003). Durante las operaciones aritméticas, el niño pasa de utilizar estrategias explícitas, como el conteo con los dedos a estrategias implícitas, que les permite realizar operaciones aritméticas mentalmente (cálculo mental), para lo cual el niño debe recuperar la información almacenada en un sistema de memoria de su cerebro (Formoso et al., 2017).

A saber, que el cálculo mental "es una serie de procedimientos mentales que se realiza sin la ayuda de papel, lápiz, calculadora o las manos y que permite obtener la respuesta exacta de problemas aritméticos" (Mochón y Vázquez, 1995, p.93). La resolución de problemas de forma eficiente durante el cálculo ha sido considerada un logro importante para que posteriormente se pueda enseñar conocimientos matemáticos de mayor complejidad (Bull, Espy y Wiebe, 2008). Varios actos cotidianos exigen la mente para realizar en forma rápida los cálculos, como, por ejemplo, deducir el vuelto de una compra o un descuento en una adquisición y otras operaciones comerciales.

Por tal motivo, es importante que el niño desarrolle el cálculo mental. El docente debe apoyarle para que consiga su propio enfoque, emplee sus propias referencias numéricas y su grado de simplificación, de tal forma que desarrolle su creatividad en los cálculos mentales en busca de la mejor opción de resolución y de forma rápida (Urquizo y Campana, 2017). Por consiguiente, el presente estudio tiene como objetivo realizar una intervención educativa extraescolar en matemáticas dirigido a niños de 7 años, con el fin de mejorar el cálculo mental, considerando que las habilidades del cálculo en los niños juegan un papel importante en el éxito académico.

\section{MATERIALES Y MÉTODOS}

\subsection{Participantes.}

La muestra estuvo conformada por 14 niños, 9 niñas y 5 niños de 7 años que se inscribieron en el curso de matemáticas en cálculo mental en la Fundación Caminos del Sol de la ciudad de Ambato. En el estudio se empleó un muestreo no probabilístico, llamado casual o accidental. Es un método de muestreo no probabilístico, debido a que los niños se eligen de manera casual, sin ningún criterio 
previo. Para la investigación se seleccionó a la Fundación Caminos de Sol por las facilidades que prestaba al estudio, y se eligieron a niños de 7 años que se encontraban matriculados en el curso de matemáticas.

\subsection{Aula de clase}

El aula donde se realizó el estudio estuvo formada por un pizarrón, un escritorio con su propia silla para la profesora de matemáticas y 14 pupitres, uno para cada niño. El curso educativo se realizó durante 10 semanas, dos horas por semana, 1 hora diaria, en los meses de mayo a julio de 2018. Como materiales se le proporcionó a cada alumno hojas con operaciones matemáticas y como instrumento de recogida de datos, una prueba de cálculo mental, que contenía 70 operaciones aritméticas combinadas entre sumas y restas.

\subsection{Actividades extraescolares.}

Las actividades extraescolares se diseñaron tomando en consideración las propuestas de enseñanza de cálculo mental de Digital Education Resource Archive (2010), nivel inicial en matemáticas (nivel I), y se efectuó de la siguiente manera:

En las primeras dos semanas se realizaron un repaso de los conocimientos de los niños de la forma de representar los números en su orden ascendente y en forma descendente, la asociación de figuras a números, y separar figuras en forma de conjuntos.

Durante la tercera a la cuarta semana, se efectuaron actividades de memorización de operaciones de sumas y restas de dos números. Para lo cual, se les entrenó a los niños en operaciones aritméticas, en forma de juego, utilizando objetos y materiales manipulables.

De la quinta a la séptima semana se les enseñó ejercicios de cálculo con tres números, primeramente, en sumas, luego en restas y finalmente en operaciones combinadas de sumas y restas. Ejemplos de operaciones aritméticas se indica en la Tabla I. Se emplearon fichas de papel con dibujos que contenían operaciones aritméticas del mismo elemento sean de objetos, frutas y animales.

De la octava a la décima semana se practicaron en cálculo mental de operaciones de tres y cuatro números (Tabla I), primeramente, en sumas, restas y luego operaciones combinadas de sumas y restas. Se emplearon fichas de papel con dibujos que contenían operaciones aritméticas. En los cálculos los niños tenían que realizarlo lo más rápidamente sin utilizar en apoyo de las manos para las operaciones.

\section{TABLA I. EJEMPLOS DE OPERACIONES} ARITMÉTICAS.

\begin{tabular}{|c|c|c|c|}
\hline \multicolumn{4}{|c|}{ Sumas y restas con dos números. } \\
\hline $2+5=7$ & $4+4=8$ & $8-2=6$ & $6-3=3$ \\
\hline $1+3=4$ & $1+5=6$ & $7-4=3$ & $10-2=8$ \\
\hline \multicolumn{4}{|c|}{ Sumas y restas con tres números. } \\
\hline $4+2+1=7$ & $9-2-4=3$ & $4-1+5=8$ & $6-3-1=2$ \\
\hline $5+2+3=10$ & $7-3-4=0$ & $6+2-4=4$ & $7-4-2=1$ \\
\hline \multicolumn{4}{|c|}{ Sumas y restas con cuatro números. } \\
\hline $3+2+2+2=9$ & $8-2-1-2=3$ & $3-1+5+1=8$ & $6-3-1+8=10$ \\
\hline $1+2+2+3=8$ & $9-3-2-4=0$ & $6+2-4-1=3$ & $7-4-3+8=8$ \\
\hline
\end{tabular}

Fuente: Tabla diseñada por autora del estudio.

\subsection{Evaluación de las actividades} extraescolares.

Con la finalidad de evaluar las actividades escolares, se empleó como instrumento de 
recogida de datos, una prueba de cálculo mental, que contenía 70 operaciones aritméticas combinadas entre sumas y restas. Cada operación aritmética poseía 4 números del 0 al 10, con un máximo de dos números negativos. Se consideró que, durante el desarrollo de los cálculos no deben sobrepasar el valor de 10, ni dar como resultado un número negativo. Ejemplo de la prueba de cálculo mental se indicada en la Tabla II.

TABLA II. PRUEBA DE CÁlCULO MENTAL EN NIÑOS.

PRUEBA: Cálculo mental

Alumno:

Fecha de realización:

Número de respuestas correctas:

Número de respuestas incorrectas:

\begin{tabular}{ccccccc}
\hline N. 1 & N.2 & N.3 & N.4 & N.5 & $\ldots \ldots \ldots$ & N. 70 \\
\hline 9 & 4 & 8 & 3 & 10 & & 3 \\
-6 & -2 & -3 & 5 & -5 & & 4 \\
-1 & 7 & 1 & -2 & -3 & & -7 \\
5 & -3 & -6 & -4 & 6 & & 5 \\
$=$ & $=$ & $=$ & $=$ & $=$ & $\ldots \ldots \ldots$ & $=$
\end{tabular}

Fuente: Tabla diseñada por autora del estudio.

En la recolección de datos, se utilizó el diseño estadístico Pretest-Postest de un solo grupo, con recolección de datos antes y después de la intervención educativa. La recolección de datos consistió en tomarles dos pruebas de cálculo mental a los niños, una antes de la intervención (Pretest) y otra al final de la intervención (Postest). En cada recogida de datos se proporcionaron una prueba diferente de cálculo, para lo cual, se les entregó a los alumnos varias hojas con 70 problemas, se les explicó la forma de llenar las respuestas en la hoja y el cálculo debían realizarse en forma mental y rápidamente. El tiempo de duración de cada prueba fue de 5 minutos.

\subsection{Análisis estadístico.}

El análisis estadístico de los datos se lo realizó utilizando el paquete estadístico Statgraphics Plus 5.1, para lo cual, las pruebas de cálculo aritmético fueron calificadas en base al número de respuestas correctas e incorrectas. Con estos datos se realizó la prueba $\mathrm{t}$ de Student, considerando al grupo de niños de la intervención educativa, como una muestra dependiente.

\section{RESULTADOS Y DISCUSIÓN}

Los resultados de las pruebas de cálculo mental en niños en Pretest (Tabla III) dieron un valor promedio de respuestas acertadas (correctas) de 16.9 \pm 4.1 , con un rango mínimo de 10 y máximo de 23 respuestas acertadas. En cambio, tienen respuestas erróneas $1.0 \pm 1.5$, en un rango de mínimo de 0 a un máximo de 4 respuestas erróneas. Existiendo 6 niños con valores menores al promedio de 16.9 respuestas acertadas. Estos valores nos indica que estos niños les falta agilidad mental para resolver los problemas aritméticos planteados en la Prueba de Pretest.

A las matemáticas se las considera como una de las áreas de la ciencia más difíciles de aprender, los profesores que acompañan el proceso de enseñanza en este campo debe ser una persona bien prepara y que sepa motivar al estudiante hacia un aprendizaje significativo de aplicación en la vida cotidiana de los niños. Según Pérez (2011) el niño necesita observar el entorno que les rodea, utilizar sus sentidos, 
manipular y experimentar con objetos. Es un trabajo arduo para el docente, que tendrá que diseñar actividades educativas que pongan en juego los sentidos de los niños; necesario para la eficiencia en la enseñanza y aprendizaje del cálculo aritmético. Considerando que, el razonamiento aritmético es posiblemente una de las habilidades cognitivas más importantes que un niño debe dominar (Rivera, Reiss, Eckert y Menon 2005).

Al final de las actividades educativas, los niños logran responder mejor en el cálculo mental con un valor promedio de $30.6 \pm 5.1$ respuestas acertadas, rango mínimo de 23 a un máximo de 39 respuestas correctas, en cambio presentaron $1.6 \pm 1.3$ respuestas erróneas con un rango de mínimo de 0 a máximo 4 de respuestas erradas.

En la Figura 1, se observa la representación del número promedio de respuestas acertadas y erróneas de las pruebas de cálculo mental en los niños de 7 años en el Pretest y Postest. En él se ve claramente que las respuestas acertadas son mayores en el Postest en comparación con el Pretest, para comprobar se realizó la prueba de t de Student (Tabla IV), encontrándose diferencia altamente significativa $(\mathrm{p}<0.01)$ entre Postest y Pretest. En cambio, en las respuestas erróneas, no existe diferencia significativa $(p<0.05)$ en las pruebas en cálculo mental en Pretes en comparación con las pruebas de cálculo Postet.

Los resultados nos indican que la intervención educativa ha mejorado la agilidad mental en la resolución de problemas aritméticos, sin embargo, presentan en promedio errores de resolución de problemas con valores de $1 \pm 1.5$ en Pretest a $1.6 \pm 1.3$ en Postest. Estadísticamente se mantiene el error en la resolución de problemas, al inicio de la intervención como al final. Estos errores, puede deberse a que se exige a los niños resolver los 70 problemas de la prueba de cálculo en 5 minutos.

TABLA III. NÚMERO DE RESPUESTAS ACERTADAS Y ERRÓNEAS DE LAS PRUEBAS DE CÁLCULO MENTAL EN NIÑOS

\begin{tabular}{|c|c|c|c|c|c|}
\hline \multirow[b]{2}{*}{ N. } & \multirow{2}{*}{ Niña/Niño } & \multicolumn{2}{|c|}{$\begin{array}{l}\text { Prueba } \\
\text { Pretest }\end{array}$} & \multicolumn{2}{|c|}{$\begin{array}{l}\text { Prueba } \\
\text { Postest }\end{array}$} \\
\hline & & $\begin{array}{c}\text { Respuestas } \\
\text { acertadas }\end{array}$ & $\begin{array}{l}\text { Respuestas } \\
\text { erróneas }\end{array}$ & $\begin{array}{c}\text { Respuestas } \\
\text { acertadas }\end{array}$ & $\begin{array}{l}\text { Respuestas } \\
\text { erróneas }\end{array}$ \\
\hline 1 & Niño & 23 & 0 & 31 & 2 \\
\hline 2 & Niño & 18 & 3 & 25 & 1 \\
\hline 3 & Niño & 13 & 2 & 26 & 1 \\
\hline 4 & Niña & 11 & 0 & 23 & 4 \\
\hline 5 & Niña & 13 & 0 & 32 & 0 \\
\hline 6 & Niña & 10 & 0 & 30 & 2 \\
\hline 7 & Niña & 19 & 0 & 35 & 0 \\
\hline 8 & Niña & 15 & 0 & 24 & 0 \\
\hline 9 & Niña & 20 & 0 & 39 & 0 \\
\hline 10 & Niña & 22 & 2 & 31 & 3 \\
\hline 11 & Niño & 14 & 0 & 26 & 2 \\
\hline 12 & Niño & 20 & 0 & 35 & 2 \\
\hline 13 & Niña & 18 & 4 & 36 & 2 \\
\hline 14 & Niña & 20 & 3 & 35 & 3 \\
\hline \multicolumn{2}{|c|}{ Promedio } & 16.9 & 1.0 & 30.6 & 1.6 \\
\hline \multicolumn{2}{|c|}{ Desviación estándar } & 4.1 & 1.5 & 5.1 & 1.3 \\
\hline \multicolumn{2}{|c|}{ Mínimo } & 10 & 0 & 23 & 0 \\
\hline \multicolumn{2}{|c|}{ Máximo } & 23 & 4 & 39 & 4 \\
\hline
\end{tabular}

Fuente: Instrumentos aplicados en el estudio. 


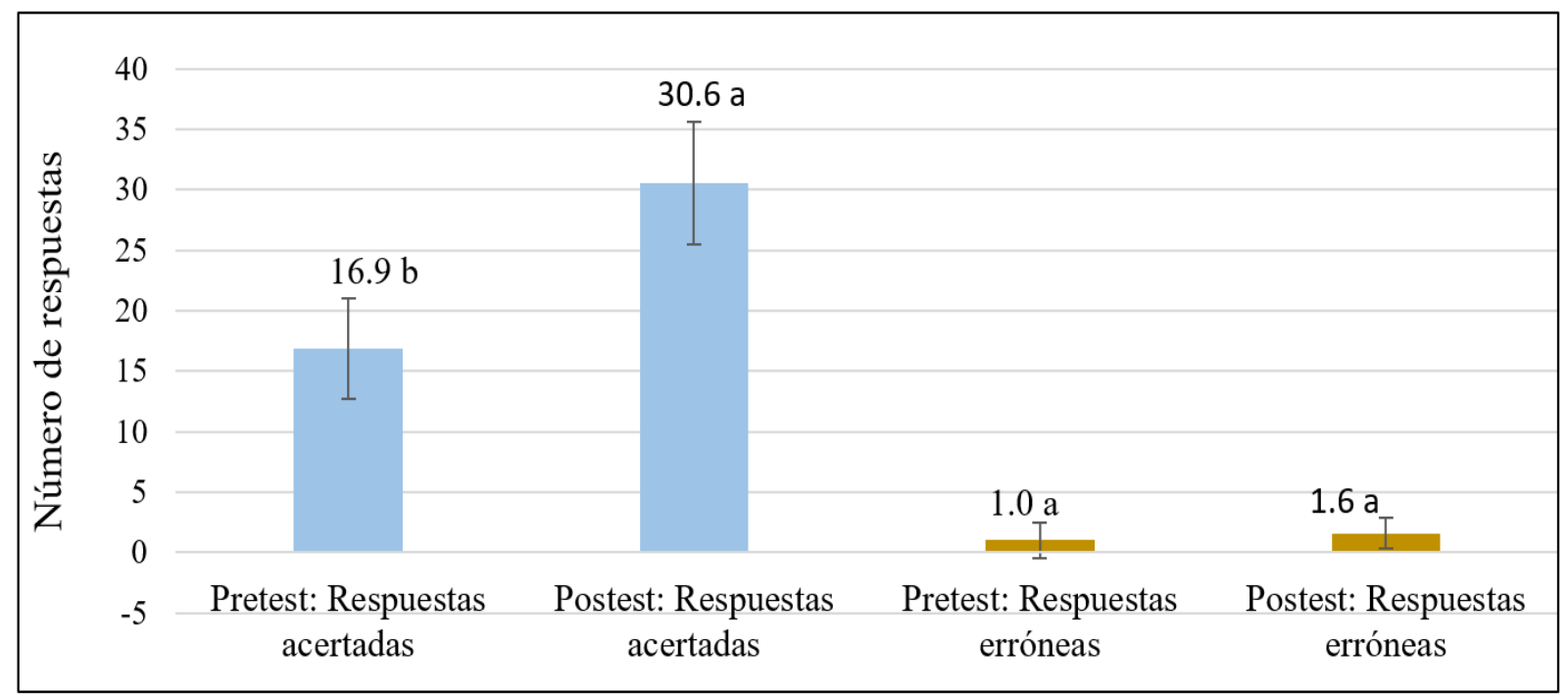

Figura 1. Promedio de respuestas acertadas y erróneas y su desviación estándar en Pretest y Postest de calculo mental. Nota: Letras diferentes indica diferencia significativa $(\mathrm{p}<0.05)$.

Fuente: Instrumentos aplicados en el estudio.

Tabla IV. Pruebas de significancia en Pretest y Postest en las respuestas de cálculo mental en niños

\begin{tabular}{llll}
\hline $\begin{array}{l}\text { Cálculo } \\
\text { mental }\end{array}$ & $\begin{array}{l}\text { Pretest } \\
\text { Promedio }\end{array}$ & $\begin{array}{l}\text { Postest } \\
\text { Promedio }\end{array}$ & $\mathrm{t}$ de Student \\
\hline $\begin{array}{l}\text { Respuestas } \\
\text { acertadas }\end{array}$ & $16.9 \pm 4.1$ & $30.6 \pm 5.1$ & $\mathrm{t}=-7.82434$ \\
$\begin{array}{l}\text { Respuestas } \\
\text { erróneas }\end{array}$ & $1.0 \pm 1.5$ & $1.6 \pm 1.3$ & $\mathrm{P}=2.67 \mathrm{E}-8^{*}$ \\
& & & $\mathrm{P}=0.282913$
\end{tabular}

Fuente: Tabla diseñada por autora del estudio.

*Diferencia altamente significativa.

En el aprendizaje de las matemáticas, los niños responden bien al uso de objetos o materiales manipulables, que ayudan a enseñar conceptos de sumas y restas, que han dado buen resultado en el cálculo mental de los niños.

Las matemáticas enseñadas a niños en los primeros niveles de educación sientan las bases, no sólo para el desarrollo del conocimiento de la misma materia, sino también para las capacidades cognitivas y las actitudes que les permitan desenvolverse adecuadamente en situaciones cotidianas (Friz, Sanhueza, Sánchez, Sánchez y Carrera, 2009).

\section{CONCLUSIONES}

En Ecuador existen problemas en el aprendizaje de las matemáticas en niños escolares, sobre todo en el cálculo aritmético. Un mal rendimiento académico en matemáticas hace que los padres de familia busquen centros de nivelación o mejoramiento académico particulares. Los centros de nivelación particulares han visto la oportunidad de crear actividades para ofrecer a la ciudadanía la oportunidad de nivelar los conocimientos a los alumnos que lo necesiten.

El presente estudio, consideró oportuno determinar si la intervención educativa en cálculo mental puede ser positivo en su aprendizaje de los niños. Por lo que podemos decir que, al final de las actividades extra extraescolares los niños de 7 años mejoraron el cálculo mental en un $80 \%$ más en el Postest con relación al Pretest. Sin embargo, mantienen el número de problemas mal resueltos o soluciones erróneas al inicio como al final de las actividades educativas. 
Estos resultados nos indican que es recomendable que los niños de 7 años que no han alcanzado las competencias básicas o presentan dificultades de aprendizaje en el cálculo aritmético y cálculo mental, puedan asistir a este tipo de actividades, que les permita entrenarse en la resolución de problemas y mejorar su aprendizaje en esta área de las matemáticas.

Aunque, se debe considerar realizar nuevos estudios que evalúen la participación con un mayor número de alumnos, esto podría ser punto de partida de futuras líneas de investigación en este campo, como, por ejemplo, continuar en la intervención educativa con la participación de un mayor número de niños y con niveles más avanzados de cálculo mental.

\section{REFERENCIAS BIBLIOGRÁFICAS}

Bull, R., Espy, K., y Wiebe, S. (2008). Shortterm memory working memory, and executive functioning in preschoolers: Longitudinal predictors of mathematical achievement at age 7 years. Developmental Neuropsychology, 33 (3), 205-228. Doi: htt ps://10.1080/87565640801982312

Digital Education Resource Archive (DERA) (2010). Teaching children to calculate mentally. Recuperado de https://dera.ioe. ac.uk/778/1/735bbb0036bed2dcdb32de11c7435b55.pdf

Flotts, M., Manzi, J., Jiménez, D., Abarzúa, A., Cayuman, C., y García, M. (2016). Informe de resultados TERCE. Terceros estudios regionales comparativos y explicativos.
Paris, Francia: Organización de las Naciones Unidas para la Educación, la Ciencia y la Cultura. Recuperado de http://unesdoc.unesco.org/images/0024/002435/243532S.pdf

Formoso, J., Injoque-Ricleab, I., Jacubovicha, S., y Barreyr, J. (2017). Cálculo mental en niños y su relación con habilidades cognitivas. Acta de Investigación Psicológica, 7(3), 2766-2774. Doi: https://10.1016/j.aipprr.2017.11.004

Friz, M., Sanhueza, S, Sánchez, A., Sánchez, M., y Carrera, C. (2009). Concepciones en la enseñanza de la matemática en educación infantil. Perfiles Educativos, 31 (125). Recuperado de http://www.scielo. org. $\mathrm{mx} /$ scielo.php?script=sci_arttext\&pi$\mathrm{d}=\mathrm{S} 0185-26982009000300005$

González, M. E., y Arencibia, R. J. (2003). El pensamiento psicológico y pedagógico de Jean Piaget. Revista de Psicología Cubana, 20(1), 87-90. Recuperado de http://pepsic.bvsalud.org/pdf/rcp/v20n1/15.pdf

Guillen, J. C. (2018). El cerebro matemático en el aula: algunas ideas clave. Recuperado de https://escuelaconcerebro.wordpress.com/ González, S.; Fernández, F., y Duarte, J. (2018). Efecto del entrenamiento de memoria de trabajo y mindfulness en la capacidad de memoria de trabajo y el desempeño matemático en niños de segundo grado. RMIE, 23(78), 841-859. Recuperado de http://www. scielo.org.mx/pdf/rmie/v23n78/1405-6666rmie-23-78-841.pdf

Melquiades, A. (2012). Estrategias didácticas para un aprendizaje constructivista en la enseñanza de las matemáticas en los niños y 
niñas de nivel primaria. Textos y Contextos, 43-58. Recuperado de https://dialnet.unirioja.es/servlet/articulo?codigo $=6349169$

Mochón, S., y Vázquez, J. (1995). Cálculo mental y estimación: Métodos, resultados de una investigación y sugerencias para su enseñanza. Educación Matemática, 7(3). Recuperado de http://www.revista-educacion-matematica.org.mx/descargas/Vol7/3/07Mochon. pdf

Moya, A. (2004). La matemática de los niños y niñas. Contribuyendo a la equidad. Revista Universitaria de Investigación, 5(29), 2336. Recuperado de http://www.redalyc.org/ pdf/410/41050203.pdf

Oficina de Educación del Condado de Sonoma (2010). Matemáticas en la casa: ayudando a sus niños a aprender y disfrutar de las matemáticas. Recuperado de https://www. scoe.org/files/math-at-home-spanish.pdf

Parviainen, P. (2019). The Development of Early Mathematical Skills - A Theoretical Framework for a Holistic Model. Journal of Early Childhood Education Research, 8(1), 019, 162-191. Recuperado de https://jecer. org/wp-content/uploads/2019/10/Parviainen-issue8-1.pdf

Penagos, M.; Mariño, L., y Hernández, R. (2017). Pensamiento matemático elemental y avanzado como actividad humana en permanente evolución. Perspectivas, 1, 105-116. Doi: https://doi.org/10.22463/25909215.1289

Pérez, C. M. (2011). Iniciación a los números en educación infantil. Pedagogía Magna, 5257. Recuperado de https://dialnet.unirioja.es/ ejemplar/277801
Poll, Y., y Guirado, V. (2017). Bases didácticas del proceso de enseñanza-aprendizaje del cálculo en los educandos de sexto grado con discapacidad intelectual leve. Educación y Sociedad, 120-132. Recuperado de http:// revistas.unica.cu/index.php/edusoc/article/ download/583/pdf

Rivera, S. M., Reiss A, L., Eckert M. A., y Menon, V. (2005). Developmental changes in mental arithmetic: Evidence for increased functional specialization in the left inferior parietal vortex. Cerebral Cortex, 15(11), 1779-1790. Doi https://doi.org/10.1093/cercor/bhi055

Urquizo, A., y Campana, A. (2017). Programa de estrategias didácticas cognitivas para el desarrollo del razonamiento matemático. Una experiencia con estudiantes de bachillerato. Boletín Redipe, 6(4). 99-111. Recuperado de https://dialnet.unirioja.es/servlet/ autor? codigo $=4324376$ 